\title{
SYNTHESIS AND BIOLOGICAL ACTIVITY OF NEW SELECTED DIFFERENT HETEROCYCLIC NITROGEN COMPOUNDS INCORPORATING 1,4-NAPHTHQUINONE
}

\author{
Ali Kamel Khalafallah \\ Department of Chemistry \\ Aswan Faculty of Science, Aswan, Egypt.
}

\begin{abstract}
:
Condensation of 1-phenylnaphthcyclopentan-2,4,9-trione II with aromatic aldehydes yielded the corresponding 3-benzylidene derivatives $\mathrm{III}_{\mathrm{af}}$ Interaction of III $_{\mathrm{a}: \mathrm{f}}$ with hydrazines, hydroxylamine, urea and thiourea afforded some new (pyrazoline $\mathrm{IV}_{\mathrm{a}-\mathrm{f}}, \mathrm{V}_{\mathrm{a}-\mathrm{f}}$, Isoxazolino $\mathrm{VI}_{\mathrm{aff}}$ pyrimidinone and/or pyrimidinethione, $\mathrm{VII}_{a-f}$. $\mathrm{VIII}_{\mathrm{a}-\mathrm{f}}$ derivatives respectively. Also, a series of some spiro compounds covering 2-lactams and thiazolidinones $\mathrm{X}_{\mathrm{g} \cdot \mathrm{j}}, \underline{\mathrm{XI}}_{\mathrm{g} \cdot \mathrm{j}}$ incorporating 1 phenylnaphthcvclopentan-2,4,9-trione were prepared.
\end{abstract}

\section{Introduction:}

An efficient strategy for synthesis of new heterocyclics via cyclocondensation reaction between different types of K,2-unsaturated ketone compounds with hydrazines, hydroxylamine, urea and thiourea. Also, this an efficient strategy extended to include a synthesis of some spiro compounds via cycloaddition reaction of monochloroacetyl chloride and thioglycolic acid to the newly synthesised Schiff bases $\mathrm{IX}_{\mathrm{b}-1}$ were the subject of our studies(1-7). In continuation to our previous work on the heterocyclic nitrogen compounds and in view of their various uses as biological and synthetic drugs

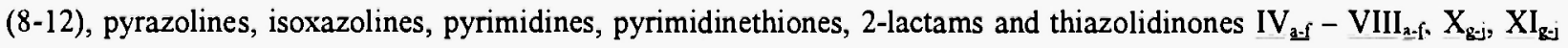
in conjunction with 1-phenylnaphthcyclopenan-4,9-dione were prepared.

\section{Results and Discussion:}

1-phenylnaphthcyclopentan-2,4,9-trione II was prepared through the reaction of 1,4-naphth- quinone with benzyl chloride in the presence of ethylene glycol as solvent and sodium bicarbonate as catalyst to give 2-benzyl-1,4naphthquinone $I$, then the cyclocondensation reaction of the previously prepared compound 1 with monochloroacetic acid proceeded in the presence of triethylamine as catalyst affording 1-phenylnaphthcyclopentan-2,4,9-trione II (Scheme 1). The structures of I and II were confirmed by the elemental analysis, IR and ${ }^{1} \mathrm{H}$ NMR spectral data (Tables 1,2 ).

Condensation of II with appropriate aromatic aldehydes proceeded smoothly in absolute alcohol using piperidine as catalyst to yield the corresponding 3-arylideno-1-phenylnaphthcyclopentan-2,4,9-trione III $_{\text {a.f. }}$.

The presence of $\mathrm{K}, 2$-unsaturated Ketonic system in compounds $\mathrm{III}_{\mathrm{a} \cdot \mathrm{f}}$ led to their reaction with hydrazines according to the reported method (4). Thus, the interaction of III $_{\mathrm{a} \cdot \mathrm{f}}$ with hydrazinehydrate in dry alcohol in the presence of glacial acetic acid afforded the corresponding 1-phenylnaphthcyclopentan-(2,3-c)-N-acetylpyrazolino-4,9-dione $\mathrm{IV}_{\text {ast }}$. However, the reaction of III $_{\text {s.f }}$ with phenyl hydrazine gave $\mathrm{N}$-phenylpyrazolino analogues $\mathrm{V}_{\mathrm{a}-\mathrm{f}}$ under the influence of piperidine catalysis 
Also, the activation exerted by the carbonyl group on the exocyclic double bond in $\underline{\mathrm{II}}_{\mathrm{aff}}$ renders them available for the addition of the various amino compounds, e.g., hydroxylamine hydrochloride, urea and thiourea. Thus, interaction of $\underline{I I}_{a-1}$ with one mole equivalent of hydroxylamine hydrochloride in ethanolic sodium hydroxide solution gave the corresponding 1-phenylnaphthcyclopentan(2,3-c)isoxazolino-4,9-dione $\underline{\mathrm{VI}}_{\mathrm{a}-\mathrm{f}}$ whereas the interaction of $\underline{\mathrm{III}}_{\mathrm{a}-\mathrm{f}}$ with equimolar ratios of urea and/or thiourea in ethanol containing hydrochloric acid gave the corresponding 1phenylnaphthcyclopentan-(2,3-c)pyrimidine(pyrimidinethione)-4,9-dione $\underline{\mathrm{Vll}}_{\mathrm{aff}}$ and $\underline{\mathrm{VIIl}}_{\mathbf{a}-\mathrm{f}}$ respectively (Scheme 1).

Structure of compounds $\underline{I-V I I I}_{\mathrm{g}-\mathrm{f}}$ were confirmed by the elemental analysis, IR and ${ }^{~} \mathrm{H}$ NMR spectral data (13-14) (Tables 1, 2).

Also 1-phenylnaphthcyclopentan-2,4,9-trione II undergo condensation reaction with different aromatic nitroso compounds such as >-nitrosophenol, >-nitroso-N-dimethylaniline, 2-nitroso-K-naphthol and K-nitroso-2-naphthol in the presence of piperidine afforded the corresponding schiff bases compounds $\underline{\mathrm{IX}}_{\mathrm{g}-\mathrm{i}}$. Its structures were confirmed by Elemental analysis, IR and 'H NMR spectra (c.f.Tables 1,2 ). This schiff bases $\underline{I X}_{\mathrm{b}-\mathrm{i}}$ reacted with chloroacetylchloride in the presence of triethylamine in DMF afforded the corresponding spiro 2-lactams $\underline{X}_{\mathrm{g}-1}$. Its structures were confirmed by Elemental analysis, IR and 'H NMR spectra (c.f. Tables 1, 2).

On the other hand the cycloaddition reaction of thioglycolic acid to the schiff bases $\underline{I X}_{R-1}$ in DMF give the

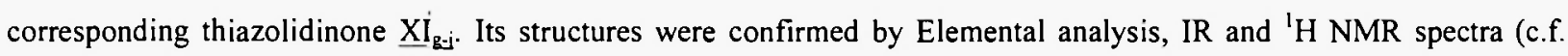
Tables 1,2).

The antibacterial and antifungal activities of some selected compounds, i.e., III-VIII glycol were determined using filter paper disc method (15) against bacteria Bacillus stearetherophil and Serratia and fungi Aspergillus and Penicillium species. The inhibition zones of all the compounds were found in the range of 6-14 mm.

Structure-biological activity relationship of the fused pyrazolines, isoxazolines and pyrimidines $\mathrm{IV}_{\underline{\mathrm{ac} . \mathrm{f}} \mathrm{f}}-\underline{\mathrm{VIII}}_{\underline{\mathrm{ac} . \mathrm{f}}}$ was demonstrated relative to the parent compound III. Thus, the parent compounds $\mathrm{III}_{\underline{a . c .} . \mathrm{f}}$ are slightly potent against bacteria and fungi. It is quite obvious that the presence of electron-donating or with drawing groups $\mathrm{III}_{\mathrm{c}}$ or $\mathrm{IIl}_{\mathrm{f}}$ increases the activity more than the unsubstituted $\mathrm{III}_{\underline{a}}$. Also, inserting a pyrazolino moiety to the parent $\underline{I I I}_{\underline{a}}$ to give $\underline{I V}_{\underline{a}}$ causes, to some extent, an increase in the biological activity. Thus, $\mathrm{N}$-acetylpyrazolino derivatives $\mathrm{IV}_{\mathrm{a} . \mathrm{c} . \mathrm{f}}$ slightly increase the biological activity, but those of $\quad \mathrm{N}$-phenylpyrazolino analogues $\underline{V}_{\mathrm{a}, c \mathrm{f}}$ increase the activity. On the other hand, insertion

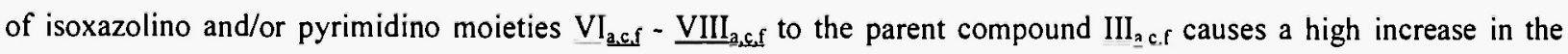
biological activity, especially those containing $>-\mathrm{N}-\left(\mathrm{CH}_{3}\right)_{2}$ substituent.

\section{Experimental:}

All melting points are uncorrected. The IR spectra were recorded on a Perkin-Elmer 127 B spectrophotometer and ${ }^{1} \mathrm{H}$ NMR spectra on an EM $390(90 \mathrm{MHz})$ spectrometer.

\section{Synthesis of 2-benzyl-1,4-naphthquinone $\underline{I}$}

A mixture of 1,4-naphthquinone $(0.01 \mathrm{~mol})$ and benzyl chloride $(0.01 \mathrm{~mol})$ in ethylene glicol $30 \mathrm{ml}$ containing 5 $\mathrm{ml}$ sodium bicarbonate $20 \%$, was refluxed for $8 \mathrm{~h}$. The hot reaction mixture was filtered; to the filtrate were added $10 \mathrm{ml}$ ethanol and a few moles of acetic acid; refluxed again for $2 \mathrm{~h}$., then to the hot reaction mixture cold water was added, whereby the product I separated out; it was filtered off, washed several times with water, dried and crystallised from the proper solvent (cf. Table 1). 


\section{Synthesis of 1-phenylnaphthcyclopentan-2,4,9-trione II}

A mixture of 2-benzyl-l,4-naphthquinone I $(0.01 \mathrm{~mol})$ and monochloro- acetic acid $(0.01 \mathrm{~mol})$ in $30 \mathrm{ml}$ ethanol containing $2 \mathrm{ml}$ triethylamine was refluxed for $10 \mathrm{~h}$. The hot reaction mixture was filtered, concentrated and boiling water was added. The product (II) precipitated out and was filtered off, washed several times with water, dried and crystallised from the proper solvent (cf. Table 1).

\section{Synthesis of 3-arylideno-1-phenylnaphthcyclopentan-2,4,9-trione $\underline{\mathrm{II}}_{\mathrm{a-f}}$}

A mixture of II $(0.01 \mathrm{~mol})$ and the aromaric aldehyde $(0.01 \mathrm{~mol})$ was dissolved in ethanol $(20 \mathrm{ml})$ containing piperidine $(1 \mathrm{ml})$ and refluxed for $25-30 \mathrm{~h}$. The reaction mixture was then filtered while hot, concentrated and allowed to cool at room temperature for overnight. On addition of petroleum ether $60-80^{\circ} \mathrm{C}$, a resinous material was separated and triturated with water. The resulting solid was filtered, washed several times with water, dried and crystallised from the proper solvent (cf. Table 1).

Synthesis of 1-phenylnaphthcyclopentan(2,3-c)- $\mathrm{N}$-acetylpyrazolino-4,9-dione $\underline{\mathrm{IV}}_{\mathbf{a}-\mathrm{f}}$.

A mixture of $\operatorname{III}_{\mathrm{a}-\mathrm{f}}(0.01 \mathrm{~mol})$ and hydrazinhydrate $(0.01 \mathrm{~mol})$ in ethanol $(20 \mathrm{ml})$ containing acetic acid $(1 \mathrm{ml})$. The reaction mixture was then filtered while hot, concentrated to one-third of its volume, poured in ice-water mixture with vigorous stirring and left overnight at room temperature. The resulting solid was filtered, washed several times with water, dried and crystallised from the proper solvent (cf. Table 1).

Synthesis of 1-phenylnaphthcyclopentan(2,3-c)-N-phenylpyrazolino-4,9-dione $\underline{V}_{\text {a-f }}$

A mixture of III $_{\mathrm{a}-\mathrm{f}}(0.01 \mathrm{~mol})$ and phenylhydrazine $(0.01 \mathrm{~mol})$ was dissolved in ethanol $(20 \mathrm{ml})$ containing piperidine $(1 \mathrm{ml})$ and refluxed for $18-26 \mathrm{~h}$. The reaction mixture was then filtered while hot, concentrated to one-third of its volume, poured in ice-water mixture with stirring for $40 \mathrm{~min}$. and left overnight at room temperature. The resulting solid was filtered, washed several times with water, dried and crystallised from the proper solvent (cf. Table 1).

\section{Synthesis of 1-phenylnaphthcyclopentan(2,3-c)isoxazolino-4,9-dione derivatives $\mathrm{VI}_{\mathrm{a}-\mathrm{f}}$}

A mixture of $\operatorname{III}_{2-\mathrm{f}}(0.01 \mathrm{~mol})$ and hydroxylamine hydrochloride $(0.01 \mathrm{~mol})$ in ethanol $(20 \mathrm{ml})$ containing $2 \%$ sodium hydroxide $(1 \mathrm{ml})$ was refluxed for $20-25 \mathrm{~h}$. The reaction mixture was then filtered while hot, the filtrate concentrated to one-third of its volume, poured in ice-water mixture with stirring for $20 \mathrm{~min}$. and left overnight at room temperature. The resulting solid was filtered, washed several times with water, dried and crystallised from the proper solvent (cf. Table 1).

\section{Synthesis of 1-phenyInaphthcyclopentan(2,3-c)pyrimidine and/or pyrimidinethione-4,9-dione derivatives} $\underline{\text { VII }}_{\text {a-f }_{\text {f }}}$ and $\underline{\text { VIII }}_{\underline{a} . f}$

A mixture of an ethanolic solution of $\operatorname{III}_{\mathrm{a}-\mathrm{f}}(0.01 \mathrm{~mol})$, urea and/or thiourea $(4 \mathrm{~g})$ and concentrated hydrochloric acid $(20 \mathrm{ml})$ was refluxed for $15-22 \mathrm{~h}$. The reaction mixture was then filtered while hot, allowed to cool and neutralised with $5 \mathrm{NaOH}$. The resulting solid was filtered, washed several times with water, dried and crystallised from the proper solvent (cf. Table 1). 


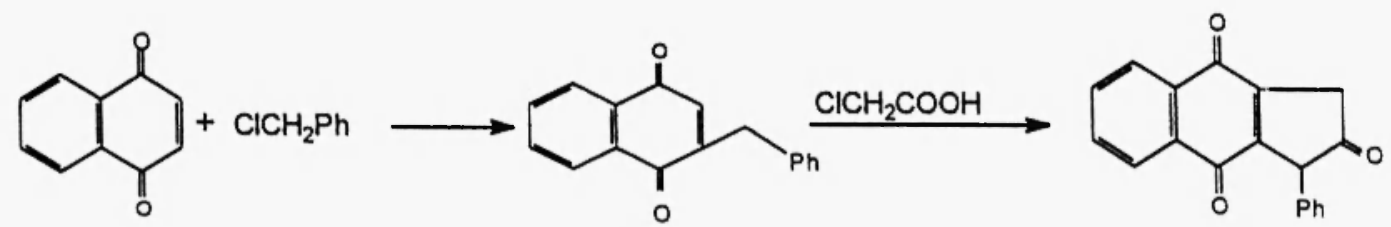

(I)

(II)

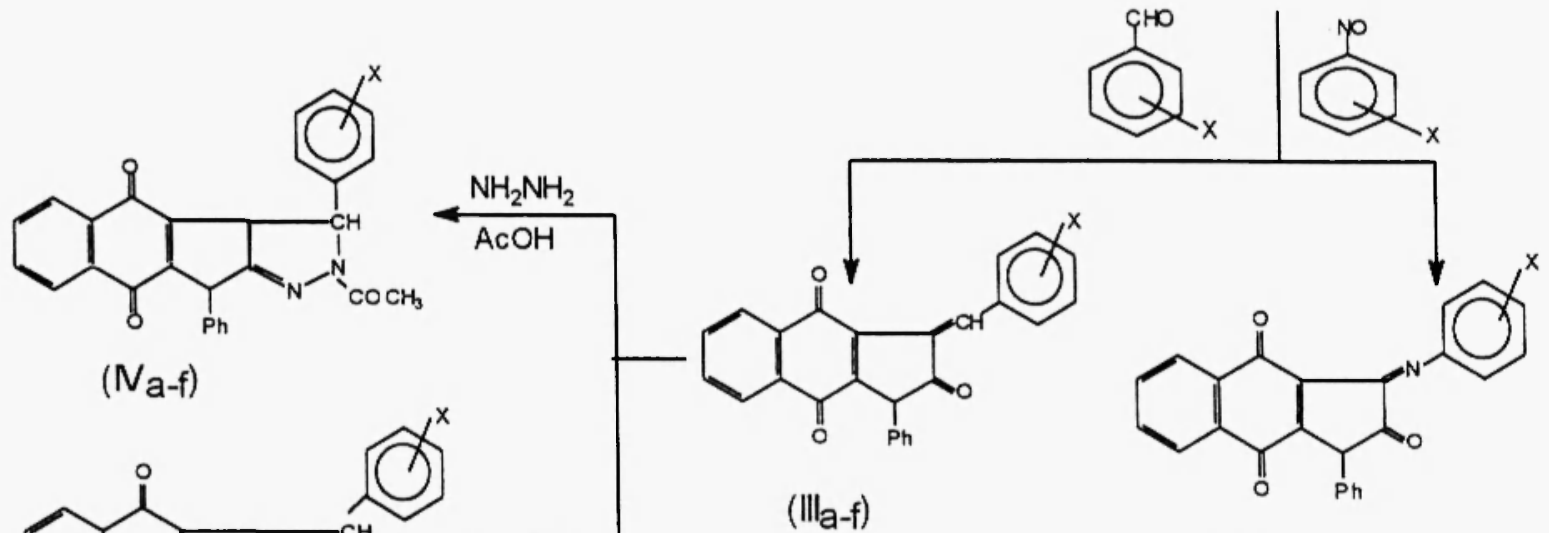

(llla-f)

piperidine

$\left(V_{a-f}\right)$

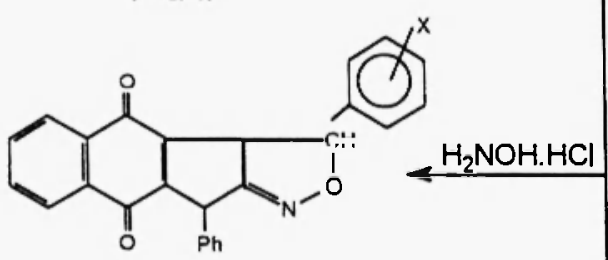

(Vla-f)<smiles>[X]c1ccc(C2NC(=O)N=C3C2C2C4C(=O)C5=C(CCCC5)C(=O)C4C(c4ccccc4)C32)cc1</smiles>

(VIla-f)

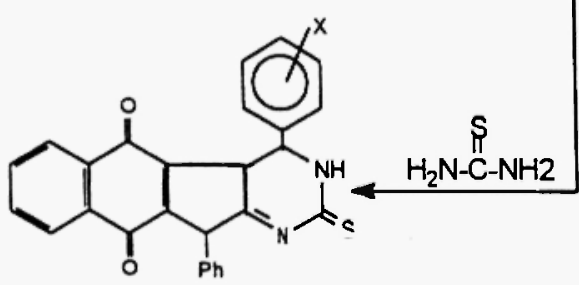

(VIIIa-f)

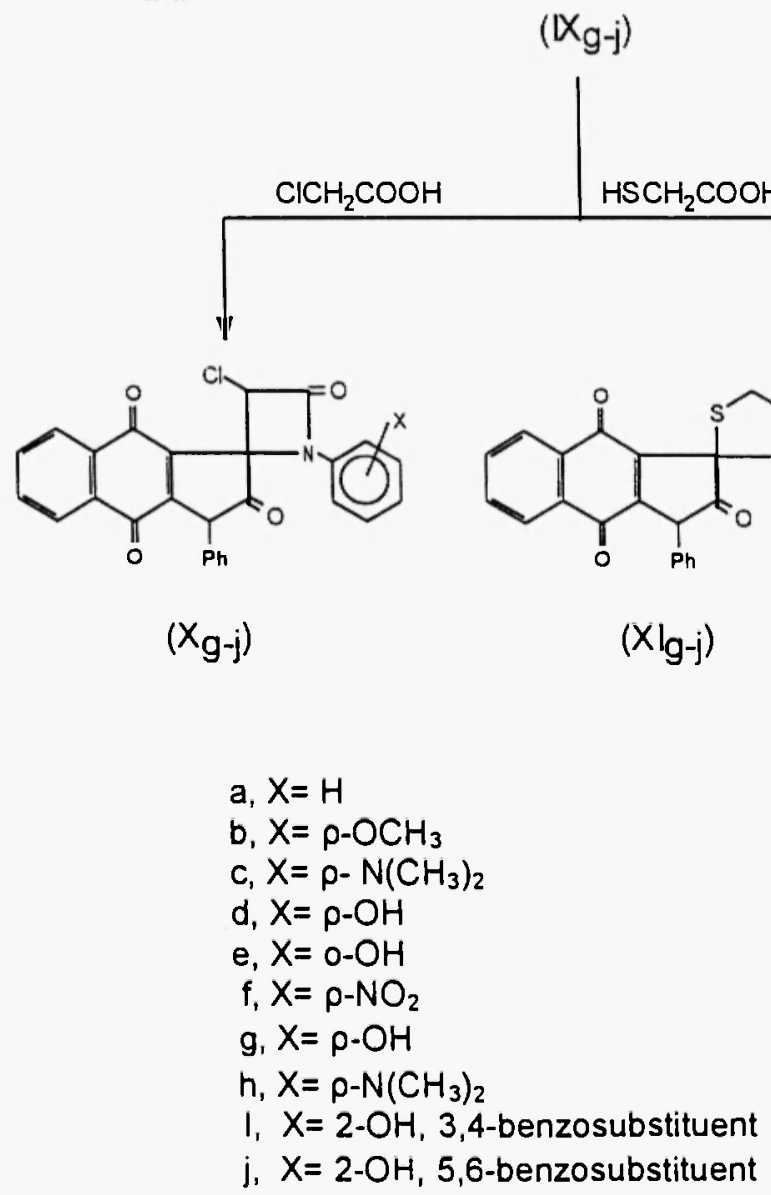

a, $X=H$

$d, X=\rho-O H$

e, $X=0-O H$

f, $X=\rho-\mathrm{NO}_{2}$

$g_{1} X=\rho-O H$

h, $X=\rho-\mathrm{N}\left(\mathrm{CH}_{3}\right)_{2}$

j, $X=2-O H, 5,6-$ benzosubstituent 
Table 1: Physical Data of Compounds I-XI

\begin{tabular}{|c|c|c|c|c|c|c|}
\hline \multirow[t]{2}{*}{$\begin{array}{l}\text { Comp. } \\
\text { No. }\end{array}$} & \multirow[t]{2}{*}{$\begin{array}{l}\text { Mol. Formula } \\
\text { (m. wt.) }\end{array}$} & \multirow[t]{2}{*}{$\begin{array}{l}\text { m.p." } \\
\left({ }^{\circ} \mathrm{C}\right)\end{array}$} & \multirow[t]{2}{*}{$\begin{array}{l}\text { Yield } \\
(\%)\end{array}$} & \multicolumn{3}{|c|}{$\begin{array}{l}\text { Elemental analysis; } \\
\text { Found (Cale.) \% }\end{array}$} \\
\hline & & & & C & $\mathbf{H}$ & $\mathbf{N}$ \\
\hline 1 & $\begin{array}{c}\mathrm{C}_{17} \mathrm{H}_{12} \mathrm{O}_{2} \\
(248) \\
\end{array}$ & $140^{\circ}$ & 60 & $\begin{array}{c}82.30 \\
(82.26) \\
\end{array}$ & $\begin{array}{c}6.90 \\
(6.85) \\
\end{array}$ & - \\
\hline II & $\begin{array}{c}\mathrm{C}_{19} \mathrm{H}_{12} \mathrm{O}_{3} \\
(288)\end{array}$ & $160^{\circ}$ & 65 & $\begin{array}{c}79.21 \\
(79.17) \\
\end{array}$ & $\begin{array}{c}4.20 \\
(4.17)\end{array}$ & - \\
\hline III $_{\text {a }}$ & $\begin{array}{c}\mathrm{C}_{26} \mathrm{H}_{16} \mathrm{O}_{3} \\
(376)\end{array}$ & $185^{\circ}$ & 55 & $\begin{array}{c}83.10 \\
(82.98)\end{array}$ & $\begin{array}{c}4.40 \\
(4.26)\end{array}$ & - \\
\hline $1 \mathrm{I}_{\mathrm{b}}$ & $\begin{array}{c}\mathrm{C}_{27} \mathrm{H}_{18} \mathrm{O}_{4} \\
(406)\end{array}$ & $205^{b}$ & 50 & $\begin{array}{c}79.90 \\
(79.80)\end{array}$ & $\begin{array}{c}4.49 \\
(4.43)\end{array}$ & - \\
\hline III $_{\mathrm{c}}$ & $\begin{array}{c}\mathrm{C}_{28} \mathrm{H}_{11} \mathrm{O}_{3} \mathrm{~N} \\
(419)\end{array}$ & $195^{b}$ & 45 & $\begin{array}{c}80.20 \\
(80.19) \\
\end{array}$ & $\begin{array}{c}5.09 \\
(5.01)\end{array}$ & $\begin{array}{c}3.39 \\
(3.34)\end{array}$ \\
\hline $\mathrm{III}_{\mathrm{d}}$ & $\begin{array}{c}\mathrm{C}_{26} \mathrm{H}_{16} \mathrm{O}_{4} \\
(392)\end{array}$ & $180^{b}$ & 52 & $\begin{array}{c}79.61 \\
(79.59)\end{array}$ & $\begin{array}{c}4.10 \\
(4.08)\end{array}$ & - \\
\hline III I $_{\mathrm{e}}$ & $\begin{array}{c}\mathrm{C}_{26} \mathrm{H}_{16} \mathrm{O}_{4} \\
(392)\end{array}$ & $230^{\circ}$ & 40 & $\begin{array}{c}80.10 \\
(79.59)\end{array}$ & $\begin{array}{c}4.48 \\
(4.08)\end{array}$ & - \\
\hline $\mathrm{III}_{\mathrm{f}}$ & $\begin{array}{c}\mathrm{C}_{26} \mathrm{H}_{15} \mathrm{NO}_{5} \\
(421)\end{array}$ & $170^{\circ}$ & 70 & $\begin{array}{c}74.20 \\
(74.11)\end{array}$ & $\begin{array}{c}3.61 \\
(3.56)\end{array}$ & $\begin{array}{c}3.40 \\
(3.33)\end{array}$ \\
\hline$I V_{a}$ & $\begin{array}{c}\mathrm{C}_{28} \mathrm{H}_{20} \mathrm{~N}_{2} \mathrm{O}_{3} \\
(432)\end{array}$ & $190^{b}$ & 60 & $\begin{array}{c}77.80 \\
(77.78)\end{array}$ & $\begin{array}{c}4.68 \\
(4.63)\end{array}$ & $\begin{array}{c}6.50 \\
(6.48)\end{array}$ \\
\hline$I V_{b}$ & $\begin{array}{c}\mathrm{C}_{29} \mathrm{H}_{22} \mathrm{~N}_{2} \mathrm{O}_{4} \\
(462)\end{array}$ & $180^{b}$ & 50 & $\begin{array}{c}75.40 \\
(75.32) \\
\end{array}$ & $\begin{array}{c}4.80 \\
(4.76)\end{array}$ & $\begin{array}{c}6.10 \\
(6.06)\end{array}$ \\
\hline$I V_{c}$ & $\begin{array}{l}\mathrm{C}_{30} \mathrm{H}_{25} \mathrm{~N}_{3} \mathrm{O}_{3} \\
\cdots \quad(475)\end{array}$ & $195^{\circ}$ & 55 & $\begin{array}{c}75.80 \\
(75.79) \\
\end{array}$ & $\begin{array}{c}5.30 \\
(5.26) \\
\end{array}$ & $\begin{array}{c}8.89 \\
(8.84) \\
\end{array}$ \\
\hline$I V_{d}$ & $\begin{array}{c}\mathrm{C}_{28} \mathrm{H}_{20} \mathrm{~N}_{2} \mathrm{O}_{4} \\
(448)\end{array}$ & $175^{b}$ & 45 & $\begin{array}{c}75.12 \\
(75.00) \\
\end{array}$ & $\begin{array}{c}4.50 \\
(4.46)\end{array}$ & $\begin{array}{c}6.30 \\
(6.25)\end{array}$ \\
\hline$I_{e}$ & $\begin{array}{c}\mathrm{C}_{28} \mathrm{H}_{19} \mathrm{~N}_{2} \mathrm{O}_{4} \\
(448)\end{array}$ & $215^{\circ}$ & 35 & $\begin{array}{c}75.45 \\
(75.00) \\
\end{array}$ & $\begin{array}{c}4.62 \\
(4.46) \\
\end{array}$ & $\begin{array}{r}6.45 \\
(6.25) \\
\end{array}$ \\
\hline$I V_{f}$ & $\begin{array}{c}\mathrm{C}_{28} \mathrm{H}_{19} \mathrm{~N}_{3} \mathrm{O}_{5} \\
(477)\end{array}$ & $170^{\circ}$ & 65 & $\begin{array}{c}70.49 \\
(70.44)\end{array}$ & $\begin{array}{c}4.10 \\
(3.98)\end{array}$ & $\begin{array}{c}8.85 \\
(8.81)\end{array}$ \\
\hline$V_{\mathrm{a}}$ & $\begin{array}{c}\mathrm{C}_{32} \mathrm{H}_{22} \mathrm{~N}_{2} \mathrm{O}_{2} \\
(466)\end{array}$ & $185^{\circ}$ & 50 & $\begin{array}{c}82.52 \\
(82.40)\end{array}$ & $\begin{array}{c}4.83 \\
(4.72)\end{array}$ & $\begin{array}{c}6.08 \\
(6.01)\end{array}$ \\
\hline$V_{b}$ & $\begin{array}{c}\mathrm{C}_{33} \mathrm{H}_{24} \mathrm{~N}_{2} \mathrm{O}_{3} \\
(496)\end{array}$ & $205^{b}$ & 40 & $\begin{array}{c}79.60 \\
(79.84)\end{array}$ & $\begin{array}{c}4.98 \\
(4.84)\end{array}$ & $\begin{array}{c}5.60 \\
(5.65)\end{array}$ \\
\hline$V_{c}$ & $\begin{array}{c}\mathrm{C}_{34} \mathrm{H}_{27} \mathrm{NO}_{2} \\
(509)\end{array}$ & $205^{b}$ & 35 & $\begin{array}{c}80.27 \\
(80.16) \\
\end{array}$ & $\begin{array}{c}5.42 \\
(5.30) \\
\end{array}$ & $\begin{array}{c}8.31 \\
(8.25) \\
\end{array}$ \\
\hline$\overline{V_{d}}$ & $\begin{array}{c}\mathrm{C}_{32} \mathrm{H}_{22} \mathrm{~N}_{2} \mathrm{O}_{3} \\
(482)\end{array}$ & $165^{b}$ & 40 & $\begin{array}{c}79.71 \\
(79.67) \\
\end{array}$ & $\begin{array}{c}4.61 \\
(4.56) \\
\end{array}$ & $\begin{array}{r}5.86 \\
(5.81) \\
\end{array}$ \\
\hline$V_{e}$ & $\begin{array}{c}\mathrm{C}_{32} \mathrm{H}_{22} \mathrm{~N}_{2} \mathrm{O}_{3} \\
(482)\end{array}$ & $220^{\circ}$ & 30 & $\begin{array}{c}79.59 \\
(79.67) \\
\end{array}$ & $\begin{array}{r}4.48 \\
(4.56) \\
\end{array}$ & $\begin{array}{c}5.92 \\
(5.81)\end{array}$ \\
\hline$v_{r}$ & $\begin{array}{c}\mathrm{C}_{32} \mathrm{H}_{21} \mathrm{~N}_{3} \mathrm{O}_{4} \\
(511)\end{array}$ & $185^{\prime}$ & 60 & $\begin{array}{c}75.20 \\
(75.15) \\
\end{array}$ & $\begin{array}{c}4.00 \\
(4.11) \\
\end{array}$ & $\begin{array}{r}8.32 \\
(8.22) \\
\end{array}$ \\
\hline $\mathrm{VI}_{2}$ & $\begin{array}{c}\mathrm{C}_{32} \mathrm{H}_{22} \mathrm{NO}_{3} \\
(468)\end{array}$ & $170^{b}$ & 55 & $\begin{array}{c}82.09 \\
(82.05)\end{array}$ & $\begin{array}{c}5.10 \\
(4.70)\end{array}$ & $\begin{array}{c}3.30 \\
(2.99)\end{array}$ \\
\hline$V I_{b}$ & $\begin{array}{c}\mathrm{C}_{33} \mathrm{H}_{24} \mathrm{NO}_{4} \\
(498)\end{array}$ & $160^{b}$ & 45 & $\begin{array}{c}79.61 \\
(79.52)\end{array}$ & $\begin{array}{c}4.91 \\
(4.82)\end{array}$ & $\begin{array}{c}2.90 \\
(2.81)\end{array}$ \\
\hline$V I_{c}$ & $\begin{array}{c}\mathrm{C}_{34} \mathrm{H}_{27} \mathrm{~N}_{2} \mathrm{O}_{3} \\
(511)\end{array}$ & $185^{\prime}$ & 35 & $\begin{array}{c}79.75 \\
(79.84) \\
\end{array}$ & $\begin{array}{r}5.19 \\
(5.28) \\
\end{array}$ & $\begin{array}{r}5.59 \\
(5.48) \\
\end{array}$ \\
\hline$V I_{d}$ & $\begin{array}{c}\mathrm{C}_{32} \mathrm{H}_{22} \mathrm{NO}_{4} \\
(484)\end{array}$ & $155^{b}$ & 40 & $\begin{array}{c}79.20 \\
(79.34) \\
\end{array}$ & $\begin{array}{c}4.41 \\
(4.55)\end{array}$ & $\begin{array}{c}2.92 \\
(2.89)\end{array}$ \\
\hline$V I_{e}$ & $\begin{array}{c}\mathrm{C}_{32} \mathrm{H}_{22} \mathrm{NO}_{4} \\
(484)\end{array}$ & $230^{\prime}$ & 30 & $\begin{array}{c}79.56 \\
(79.34) \\
\end{array}$ & $\begin{array}{c}4.67 \\
(4.55) \\
\end{array}$ & $\begin{array}{r}2.94 \\
(2.89) \\
\end{array}$ \\
\hline
\end{tabular}


Table 1: Physical Data of Compounds I-XI (continue)

\begin{tabular}{|c|c|c|c|c|c|c|c|}
\hline \multirow[t]{2}{*}{$\begin{array}{l}\text { Comp. } \\
\text { No. }\end{array}$} & \multirow[t]{2}{*}{$\begin{array}{l}\text { Mol. Formula } \\
\text { (m. wt.) }\end{array}$} & \multirow[t]{2}{*}{$\begin{array}{l}\text { m.p." } \\
\left({ }^{*} \mathrm{C}\right)\end{array}$} & \multirow[t]{2}{*}{$\begin{array}{l}\text { Yield } \\
(\%)\end{array}$} & \multicolumn{4}{|c|}{$\begin{array}{l}\text { Elemental analysis; } \\
\text { Found (Calc.) \% }\end{array}$} \\
\hline & & & & $\bar{C}$ & $\mathrm{H}$ & $\mathbf{N}$ & $S$ \\
\hline $\mathrm{VI}_{\mathrm{f}}$ & $\begin{array}{c}\mathrm{C}_{32} \mathrm{H}_{21} \mathrm{~N}_{2} \mathrm{O}_{5} \\
(513)\end{array}$ & $150^{\circ}$ & 70 & $\begin{array}{c}74.90 \\
(74.85) \\
\end{array}$ & $\begin{array}{c}4.14 \\
(4.09) \\
\end{array}$ & $\begin{array}{c}5.51 \\
(5.46) \\
\end{array}$ & - \\
\hline VII. & $\begin{array}{c}\mathrm{C}_{27} \mathrm{H}_{18} \mathrm{~N}_{2} \mathrm{O}_{3} \\
(418)\end{array}$ & $185^{\circ}$ & 50 & $\begin{array}{c}77.60 \\
(77.51)\end{array}$ & $\begin{array}{c}4.41 \\
(4.31)\end{array}$ & $\begin{array}{c}6.72 \\
(6.69) \\
\end{array}$ & - \\
\hline $\mathrm{VII}_{\mathrm{v}}$ & $\begin{array}{c}\mathrm{C}_{28} \mathrm{H}_{20} \mathrm{~N}_{2} \mathrm{O}_{4} \\
(448)\end{array}$ & $175^{2}$ & 40 & $\begin{array}{c}75.10 \\
(75.00)\end{array}$ & $\begin{array}{c}4.56 \\
(4.46) \\
\end{array}$ & $\begin{array}{c}6.45 \\
(6.25) \\
\end{array}$ & - \\
\hline$V^{\prime} I_{c}$ & $\begin{array}{c}\mathrm{C}_{29} \mathrm{H}_{23} \mathrm{~N}_{3} \mathrm{O}_{3} \\
(461)\end{array}$ & $190^{b}$ & 35 & $\begin{array}{c}75.39 \\
(75.49) \\
\end{array}$ & $\begin{array}{c}4.87 \\
(4.98) \\
\end{array}$ & $\begin{array}{c}9.21 \\
(9.11) \\
\end{array}$ & - \\
\hline $\mathrm{VII}_{\mathrm{d}}$ & $\begin{array}{c}\mathrm{C}_{27} \mathrm{H}_{18} \mathrm{~N}_{2} \mathrm{O}_{4} \\
(434)\end{array}$ & $180^{6}$ & 30 & $\begin{array}{c}74.76 \\
(74.65) \\
\end{array}$ & $\begin{array}{c}4.20 \\
(4.15) \\
\end{array}$ & $\begin{array}{c}6.51 \\
(6.45) \\
\end{array}$ & - \\
\hline $\mathrm{VII}_{\mathrm{e}}$ & $\begin{array}{c}\mathrm{C}_{27} \mathrm{H}_{18} \mathrm{~N}_{2} \mathrm{O}_{4} \\
(434)\end{array}$ & $225^{2}$ & 45 & $\begin{array}{c}74.70 \\
(74.65) \\
\end{array}$ & $\begin{array}{c}4.21 \\
(4.15) \\
\end{array}$ & $\begin{array}{c}6.57 \\
(6.45) \\
\end{array}$ & - \\
\hline$V 1 l_{r}$ & $\begin{array}{c}\mathrm{C}_{27} \mathrm{H}_{17} \mathrm{~N}_{3} \mathrm{O}_{5} \\
(463)\end{array}$ & $195^{b}$ & 60 & $\begin{array}{c}70.10 \\
(69.98)\end{array}$ & $\begin{array}{c}3.80 \\
(3.67)\end{array}$ & $\begin{array}{c}9.08 \\
(9.07)\end{array}$ & - \\
\hline VIII $_{\imath}$ & $\begin{array}{c}\mathrm{C}_{27} \mathrm{H}_{18} \mathrm{~N}_{2} \mathrm{O}_{2} \mathrm{~S} \\
(\mathbf{4 3 4})\end{array}$ & $170^{\circ}$ & 50 & $\begin{array}{r}74.85 \\
(74.65) \\
\end{array}$ & $\begin{array}{c}4.35 \\
(4.15) \\
\end{array}$ & $\begin{array}{r}6.59 \\
(6.45) \\
\end{array}$ & - \\
\hline VIII $_{b}$ & $\begin{array}{c}\mathrm{C}_{28} \mathrm{H}_{20} \mathrm{~N}_{2} \mathrm{O}_{3} \mathrm{~S} \\
(464)\end{array}$ & $185^{2}$ & 40 & $\begin{array}{c}72.51 \\
(72.41) \\
\end{array}$ & $\begin{array}{c}4.42 \\
(4.31) \\
\end{array}$ & $\begin{array}{c}6.07 \\
(6.03) \\
\end{array}$ & - \\
\hline $\mathrm{VIII}_{\mathrm{c}}$ & $\begin{array}{c}\mathrm{C}_{29} \mathrm{H}_{23} \mathrm{~N}_{3} \mathrm{O}_{2} \mathrm{~S} \\
(477)\end{array}$ & $165^{2}$ & 30 & $\begin{array}{r}73.10 \\
(72.96) \\
\end{array}$ & $\begin{array}{c}5.10 \\
(4.82)\end{array}$ & $\begin{array}{c}9.10 \\
(8.81)\end{array}$ & - \\
\hline VIII $_{d}$ & $\begin{array}{c}\mathrm{C}_{27} \mathrm{H}_{18} \mathrm{~N}_{2} \mathrm{O}_{3} \mathrm{~S} \\
\cdots(450)\end{array}$ & $170^{\circ}$ & 45 & $\begin{array}{c}72.10 \\
(72.00)\end{array}$ & $\begin{array}{c}4.14 \\
(4.00)\end{array}$ & $\begin{array}{c}6.31 \\
(6.22)\end{array}$ & - \\
\hline VIII $_{e}$ & $\begin{array}{c}\mathrm{C}_{27} \mathrm{H}_{18} \mathrm{~N}_{2} \mathrm{O}_{3} \mathrm{~S} \\
(450)\end{array}$ & $230^{6}$ & 30 & $\begin{array}{c}72.50 \\
(72.00) \\
\end{array}$ & $\begin{array}{c}4.53 \\
(4.00) \\
\end{array}$ & $\begin{array}{c}6.50 \\
(6.22) \\
\end{array}$ & - \\
\hline $\mathrm{VIII}_{r}$ & $\begin{array}{c}\mathrm{C}_{27} \mathrm{H}_{17} \mathrm{~N}_{3} \mathrm{O}_{4} \mathrm{~S} \\
(479)\end{array}$ & $150^{6}$ & 70 & $\begin{array}{c}67.68 \\
(67.64) \\
\end{array}$ & $\begin{array}{c}3.59 \\
(3.55) \\
\end{array}$ & $\begin{array}{r}8.91 \\
(8.77) \\
\end{array}$ & - \\
\hline IXg & $\begin{array}{c}\mathrm{C}_{25} \mathrm{H}_{15} \mathrm{NO}_{4} \\
(393.40)\end{array}$ & 215 & 60 & $\begin{array}{c}76.35 \\
(76.33)\end{array}$ & $\begin{array}{c}3.85 \\
(3.84)\end{array}$ & $\begin{array}{c}3.58 \\
(3.56)\end{array}$ & - \\
\hline IXh & $\begin{array}{c}\mathrm{C}_{27} \mathrm{H}_{20} \mathrm{~N}_{2} \mathrm{O}_{3} \\
(420.47) \\
\end{array}$ & 225 & 65 & $\begin{array}{c}77.14 \\
(77.13) \\
\end{array}$ & $\begin{array}{c}4.80 \\
(4.79) \\
\end{array}$ & $\begin{array}{c}6.86 \\
(6.66) \\
\end{array}$ & - \\
\hline $\mathbf{I X i}$ & $\begin{array}{c}\mathrm{C}_{29} \mathrm{H}_{17} \mathrm{NO}_{3} \\
(427.46)\end{array}$ & 240 & 67 & $\begin{array}{c}81.50 \\
(81.49) \\
\end{array}$ & $\begin{array}{c}4.03 \\
(4.01) \\
\end{array}$ & $\begin{array}{c}3.29 \\
(3.28) \\
\end{array}$ & - \\
\hline IXj & $\begin{array}{l}\mathrm{C}_{29} \mathrm{H}_{17} \mathrm{NO}_{3} \\
(427.46)\end{array}$ & 250 & 69 & $\begin{array}{c}81.51 \\
(81.49) \\
\end{array}$ & $\begin{array}{r}4.02 \\
(4.01) \\
\end{array}$ & $\begin{array}{c}3.20 \\
(3.28) \\
\end{array}$ & - \\
\hline $\mathrm{Xg}$ & $\begin{array}{c}\mathrm{C}_{27} \mathrm{H}_{16} \mathrm{NO}_{5} \mathrm{Cl} \\
(469.89)\end{array}$ & 230 & 68 & $\begin{array}{c}69.03 \\
(69.02) \\
\end{array}$ & $\begin{array}{c}3.45 \\
(3.43)\end{array}$ & $\begin{array}{c}2.99 \\
(2.98)\end{array}$ & - \\
\hline$\overline{X h}$ & $\begin{array}{c}\mathrm{C}_{29} \mathrm{H}_{21} \mathrm{~N}_{2} \mathrm{O}_{4} \mathrm{CI} \\
(496.96) \\
\end{array}$ & 245 & 60 & $\begin{array}{c}70.10 \\
(70.09) \\
\end{array}$ & $\begin{array}{c}4.27 \\
(4.26) \\
\end{array}$ & $\begin{array}{c}5.66 \\
(5.64) \\
\end{array}$ & - \\
\hline $\mathbf{X i}$ & $\begin{array}{c}\mathrm{C}_{31} \mathrm{H}_{18} \mathrm{NO}_{4} \mathrm{Cl} \\
(503.95)\end{array}$ & 260 & 55 & $\begin{array}{c}73.89 \\
(73.88) \\
\end{array}$ & $\begin{array}{c}3.62 \\
(3.60) \\
\end{array}$ & $\begin{array}{c}2.79 \\
(2.78) \\
\end{array}$ & - \\
\hline $\mathbf{X j}$ & $\begin{array}{c}\mathrm{C}_{31} \mathrm{H}_{18} \mathrm{NO}_{4} \mathrm{Cl} \\
(503.95) \\
\end{array}$ & 280 & 67 & $\begin{array}{c}73.90 \\
(73.88) \\
\end{array}$ & $\begin{array}{c}3.63 \\
(3.60) \\
\end{array}$ & $\begin{array}{c}2.80 \\
(2.78) \\
\end{array}$ & - \\
\hline XIg & $\begin{array}{c}\mathrm{C}_{27} \mathrm{H}_{17} \mathrm{NO}_{5} \mathrm{~S} \\
(467.50) \\
\end{array}$ & 265 & 69 & $\begin{array}{c}69.39 \\
(69.37) \\
\end{array}$ & $\begin{array}{c}3.68 \\
(3.67) \\
\end{array}$ & $\begin{array}{c}3.00 \\
(2.96) \\
\end{array}$ & $\begin{array}{c}6.88 \\
(6.86) \\
\end{array}$ \\
\hline XIh & $\begin{array}{c}\mathrm{C}_{29} \mathrm{H}_{22} \mathrm{~N}_{2} \mathrm{O}_{4} \mathrm{~S} \\
(494.56)\end{array}$ & 255 & 70 & $\begin{array}{c}70.45 \\
(70.43)\end{array}$ & $\begin{array}{c}4.49 \\
(4.48)\end{array}$ & $\begin{array}{c}5.67 \\
(5.66)\end{array}$ & $\begin{array}{c}6.49 \\
(6.48)\end{array}$ \\
\hline$\overline{X I i}$ & $\begin{array}{c}\mathrm{C}_{33} \mathrm{H}_{19} \mathrm{NO}_{4} \mathrm{~S} \\
(501.56)\end{array}$ & 270 & 65 & $\begin{array}{c}74.25 \\
(74.24) \\
\end{array}$ & $\begin{array}{c}3.83 \\
(3.82) \\
\end{array}$ & $\begin{array}{c}3.00 \\
(2.79) \\
\end{array}$ & $\begin{array}{r}6.41 \\
(6.39)\end{array}$ \\
\hline$X I j$ & $\begin{array}{c}\mathrm{C}_{31} \mathrm{H}_{19} \mathrm{NO}_{4} \mathrm{~S} \\
(501.56)\end{array}$ & 285 & 60 & $\begin{array}{c}74.26 \\
(74.24) \\
\end{array}$ & $\begin{array}{c}3.84 \\
(3.82) \\
\end{array}$ & $\begin{array}{c}3.01 \\
(2.79) \\
\end{array}$ & $\begin{array}{r}6.41 \\
(6.39) \\
\end{array}$ \\
\hline
\end{tabular}

Solvent for crystallisation: $a=$ ethanol; $b=$ methanol. 
Table 2: IR and ${ }^{1} \mathrm{H}$ NMR spectral data of some selected Heterocyclic Compounds $\underline{\mathrm{I}-\mathrm{XI}}$

\begin{tabular}{|c|c|c|}
\hline $\begin{array}{l}\text { Comp. } \\
\text { No. }\end{array}$ & IR $\left(\mathrm{KBr} ; \max \mathrm{cm}^{-1}\right)$ & ${ }^{T}$ H NMR (DMSO) ppm \\
\hline I & $1720(\mathrm{C}=\mathrm{O})$ & 7.06-8.0 (m,9H, aromatic), $3.22\left(\mathrm{~s}, 2 \mathrm{H},-\mathrm{CH}_{2}\right.$ joined to phenyl). \\
\hline II & $1735(\mathrm{C}=\mathrm{O})$ & $\begin{array}{l}\text { 7.06-8.0 (m,9H, aromatic), } 4.32(\mathrm{~s}, 1 \mathrm{H},-\mathrm{CH} \text { joined to phenyl }) \\
3.12\left(\mathrm{~s}, 2 \mathrm{H},-\mathrm{CH}_{2} \text { cyclopentanone). }\right.\end{array}$ \\
\hline III $_{\text {: }}$ & $\begin{array}{l}1735(\mathrm{C}=\mathrm{O}) \\
1610(\mathrm{C}=\mathrm{C})\end{array}$ & $\begin{array}{l}\text { 7.06-8.0 (m,14H, aromatic }), 4.8(\mathrm{~s}, 1 \mathrm{H}, \text { ylidene }) \\
\text { 4.32(s, } 1 \mathrm{H}, \mathrm{CH} \text { joined to phenyl). }\end{array}$ \\
\hline$I V_{\mathrm{a}}$ & $\begin{array}{l}1745(\mathrm{C}=\mathrm{O}), \\
1575-1520(\mathrm{C}=\mathrm{N})\end{array}$ & $\begin{array}{l}\text { 7.06-8.0 (m,14H, aromatic), } 2.2\left(\mathrm{~s}, 3 \mathrm{H},-\mathrm{CH}-\mathrm{CH}_{3}\right) \text {, } \\
\text { 7.1-7.3 (d, } 2 \mathrm{H} \text {, pyrazoline), } 4.32(\mathrm{~s}, 1 \mathrm{H},-\mathrm{CH} \text { joined to phenyl). }\end{array}$ \\
\hline$V_{\mathrm{a}}$ & $\begin{array}{l}1775(\mathrm{C}=\mathrm{O}) \\
1575(\mathrm{C}=\mathrm{N}\end{array}$ & $\begin{array}{l}\text { 7.06-8.0 (m,14H, aromatic),7.2-7.5(d, } 2 \mathrm{H}, \text { pyrazoli- ne protons }) \text {, } \\
4.32(\mathrm{~s}, 1 \mathrm{H},-\mathrm{CH},-\mathrm{CH} \text { joined to phenyl). }\end{array}$ \\
\hline $\mathrm{VI}_{\mathrm{a}}$ & $\begin{array}{l}1745(\mathrm{C}=\mathrm{O}) \\
1575(\mathrm{C}=\mathrm{N})\end{array}$ & $\begin{array}{l}\text { 7.06-8.0 (m,14H, aromatic), } 6.6-6.8(\mathrm{~d}, 2 \mathrm{H} \text {, isoxazol- ine protons), } \\
4.32(\mathrm{~s}, 1 \mathrm{H},-\mathrm{CH} \text { joined to phenyl). }\end{array}$ \\
\hline $\mathrm{VII}_{\mathrm{a}}$ & $\begin{array}{l}1735(\mathrm{C}=\mathrm{O}) \\
1575(\mathrm{C}=\mathrm{N}) \\
3400-3300(\mathrm{~N}-\mathrm{H})\end{array}$ & $\begin{array}{l}\text { 7.06-8.0 (m,14H, aromatic), } 3.1(\mathrm{~b}, 1 \mathrm{H},-\mathrm{NH}) \text {, } \\
6.4-6.6(\mathrm{~m}, 2 \mathrm{H}, \text { pyrimidine protons }), 4.32(\mathrm{~s}, 1 \mathrm{H},-\mathrm{CH} \text { joined to phenyl). }\end{array}$ \\
\hline $\mathrm{VIII}_{\mathrm{a}}$ & $\begin{array}{l}1700(\mathrm{C}=\mathrm{O}) \\
1575(\mathrm{C}=\mathrm{N}) \\
3400(\mathrm{~N}-\mathrm{H})\end{array}$ & $\begin{array}{l}\text { 7.06-8.0 }(\mathrm{m}, 14 \mathrm{H}, \text { aromatic }), 3.1(\mathrm{~b}, 1 \mathrm{H},-\mathrm{NH}) \\
\text { 5.4-5.6 }(\mathrm{m}, 2 \mathrm{H}, \text { pyrimidine thiono protons }) \\
\text { 4.32 }(\mathrm{s}, 1 \mathrm{H},-\mathrm{CH} \text { joined to phenyl). }\end{array}$ \\
\hline$\overline{\mathbf{I X g}}$ & $\begin{array}{l}1735(\mathrm{C}=\mathrm{O}) \\
1580(\mathrm{C}=\mathrm{N})\end{array}$ & 7.01-8.2 (m,14H, aromatic), $4.32(\mathrm{~s}, 1 \mathrm{H},-\mathrm{CH}$ joined to phenyl). \\
\hline $\mathrm{Xg}$ & $1745(\mathrm{C}=\mathrm{O})$ & $7.02-8.1(\mathrm{~m}, 14 \mathrm{H}$, aromatic $), 1.4(\mathrm{~s}, 1 \mathrm{H})$ \\
\hline $\mathrm{Xlg}$ & $1735(\mathrm{C}=\mathrm{O})$ & 7.03-8.1(m,14H, aromatic), $1.2(\mathrm{~s}, \mathrm{CH}$ of thiazolidinone). \\
\hline
\end{tabular}




\section{Synthesis of 3-azoarylmethylene-1-phenyl-2,4,9-trione derivatives $\underline{I X}_{\underline{m-i}}$}

A solution of (II) $(0.01 \mathrm{~mole})$ in ethanol $(30 \mathrm{ml})$ was treated with aromatic nitroso compounds $(0.01 \mathrm{~mole})$ in the presence of catalytic amount of piperidine $(0.5 \mathrm{ml})$. The reaction mixture was heated under reflux for 7-9 hr. (monitored by $T L C$ ). The solvent was then evaporated under reduced pressure and the residue was treated with ice/water. The solid product was collected and crystallized from ethanol.

Synthesis of 3-spiro(chloro-N-substituted-2-lactam)-I-phenyl-2,4,9-trione derivatives $\underline{X}_{-\cdot j}$ and 3-spyroNsubstituted thiazolidinone-1-phenyl-2,4,9-trione derivatives $\underline{X I}_{\boldsymbol{g}_{-j}}$

A solution of (IXg-j) was treated with chloroacetylchloride or mercaptoacetic acid in DMF $(30 \mathrm{ml})$ in the presence of catalytic amount of triethylamine $(0.1 \mathrm{ml})$. The The reaction mixture was heated under reflux for $8-10 \mathrm{hr}$. (monitored by TLC). The solvent was then evaporated under reduced pressure and the residue was treated with ice/water. The solid product was collected and crystallized from DMF.

\section{References:}

(1) A. K. Khalafallah, F. M. Abd El-Latif, M. A. Salim and M. A. El-Maghraby, Asian J. Chem., 5, 988 (1993).

(2) A. K. Khalafallah, A. I. M. Koraiem, M. A. El-Maghraby, and H. A. Shindy, J. Indian Chem. Soc., 66, 398 (1989).

(3) M. A. El-Maghraby, A. K. Khalafallah, M. E. Hassan and H. A. soliman, J. Indian Chem. Soc., 63, 910 (1986).

(4) A. K. Khalafallah, A. Abou El-Ela, E. Elshami and M. A. El-Maghraby, J. Indian Chem. Soc., 62, 676 (1985).

(5) M. A. El-Maghraby, A. I. M. Koraiem, A. K. Khalafallah, Asw. Sci. Tech. Bull., 5, I (1984).

(6) A. K. Khalafallah, M. A. Selim, R. M. Abu El-hamd, M. A. El-Maghraby, H. A. Soleiman and M. A. Raslan, Indian J. of Chem., Vol. 34B, December 1995, pp. 1066-1070.

(7) H. A. Soleiman, A. K. Khalafallah and H. M. Abd El-zaher, J. Chine. Chem. Soc., Vol. 47, pp. 1267-1272 (2000).

(8) P. P. Gillman, R. J. Belly, T. K. Kosselok and S. Zigman, U. S. 4, 323, 121 (1980); Chem. Abstr., 94, 97109 (1981).

(9) Y. Makisoumi, Japan, 13, 641 (1960); Chem. Abstr., 60, 531 (1964).

(10) A. Takamizowa and Y. Mamoshima, Japan, 14423 (1963); Chem. Abstr., 65, 20144 (1966).

(11) 1. Ito, Japan, 7030, 10 (1970); Chem. Abstr., 74, 22827 (1971).

(12) A. Takamizowa and Sato, Japan, 72, 45353 (1972) Chem. Abstr., 78, 58454 (1973).

(13) L. J. Bellamy, The Infrared Spectra of Complex Molecules, $2^{\text {nd }}$ Edn., Methuen, London, (1964).

(14) F. Scheimann, Nuclear Magnetic Resonance and Infrared Spectroscopy, Vol. 1, pp. 41-70 (1970).

(15) Y. H. Loo, P. S. Skell, H. H. Thorabrry, J. L. Megurire, G. M. Savage and J. C. Sylvester, J. Bact., 50, 701 (1945).

\section{Received on May 26, 2001}

